\title{
Simultaneous absorption of two gases in a reactive lhquid, one gas reacting instantaneously
}

(Received 7 December 1976, accepted 23 March 1977)

The case of simultaneous absorption and reaction of gases in a reactive medium, in which one of the gases reacts instantaneously, is of considerable industrial importance A typical example is the selective removal of hydrogen sulfide out of gases, also containing carbondioxide, by means of a solution of alkanol amines[10] Hydrogen sulfide reacts in the liquid instantaneously, carbondioxide has a finite rate

Several authors [2-6] have dealt with the problem of finding expressions for the simultaneous mass transfer A number of solutions are avalable, but they are all implicit or numerical

In this contribution an explicit solution is added and a comparison is made between the different models

\section{REACTION KINETICS AND MASS TRANSFER MODEL}

We consider the simultaneous absorption of the gas phase components $A$ and $B$ in a hquid containing a non-volatile reagent $C$ The reactions proceed according to

$$
\begin{aligned}
& A+z_{a} C \rightarrow \text { Producls } \\
& B+z_{b} C \rightarrow \text { Products }
\end{aligned}
$$

The reaction rate of both reactions is of second order (first order in each component), with that of $A$ being infinitely fast

A filmmodel of this system (Fig 1) is extensively described by Goettler et al [2], Ouwerkerk [5] and Ramachandran et al [6] and will only be summarized here According to Ramachandran [6] the film model gives (in dimensionless notation)

$$
\begin{array}{ll}
0<\xi<\xi_{p} & \frac{\mathrm{d}^{2} a}{\mathrm{~d} \xi^{2}}=\frac{\mathrm{d}^{2} b}{\mathrm{~d} \xi^{2}}=0 \\
\xi_{\mathrm{p}}<\xi<1 & \frac{\mathrm{d}^{2} c}{\mathrm{~d} \xi^{2}}=\frac{\mathrm{d}^{2} b}{\mathrm{~d} \xi^{2}}=b c M_{b}
\end{array}
$$

with the boundary conditions

$$
\begin{aligned}
& \xi=0 \quad a=a_{i}, b=b_{t} \\
& \xi=\xi_{p} \quad a=0, b=b_{\lambda}, c=0 \\
& \xi=1 \quad a=b=0, c=1
\end{aligned}
$$

The relationship between the enhancement factors for mass transfer of $a$ and $b, \phi_{a}$ and $\phi_{b}$, respectively, and the various fluxes are

$$
\begin{gathered}
-\left.\frac{\mathrm{d} a}{\mathrm{~d} \xi}\right|_{\xi=\xi_{\mathrm{p}}}=\left.\frac{\mathrm{d} c}{\mathrm{~d} \xi}\right|_{\xi-\xi_{p}} \\
\left.\frac{\mathrm{d} c}{\mathrm{~d} \xi}\right|_{\xi=1}=\phi_{b} b_{\mathrm{t}}+\phi_{a} a_{1} \\
\phi_{a}=-\left.\left(\frac{\mathrm{d} a}{\mathrm{~d} \xi} / a_{1}\right)\right|_{\xi=0} \\
\phi_{b}=-\left.\left(\frac{\mathrm{d} b}{\mathrm{~d} \xi} / b_{\mathrm{l}}\right)\right|_{\xi=0}
\end{gathered}
$$

where

$$
h=\frac{Z_{B} D_{b} B}{D_{c} C_{0}}, \quad b_{\imath}=\frac{z_{b} D_{b} B_{1}}{D_{c} C_{0}}, \quad b_{\lambda}=\frac{z_{b} D_{b} B_{\lambda}}{D_{c} C_{0}}
$$

$$
\begin{gathered}
a=\frac{z_{a} D_{a} A}{D_{c} C_{0}}, \quad a_{a}=\frac{z_{a} D_{a} A_{i}}{D_{c} C_{0}} \\
c=\frac{C}{C_{0}} \\
\xi=\frac{x}{\delta}, \quad \xi_{p}=\frac{\lambda}{\delta} \\
\delta=\frac{D_{b}}{k_{\mathrm{L}}} \\
M_{b}=k_{b} D_{b} C_{0} / k_{\mathbf{L}}{ }^{2}
\end{gathered}
$$

The system can be transformed into

$$
\xi_{p}<\xi<1 \frac{\mathrm{d}^{2} b}{\mathrm{~d} \xi^{2}}=b c M_{b}
$$

with the boundary conditions

$$
\begin{gathered}
c\left(\xi_{p}\right)=0 \\
c(1)=1 \\
\phi_{b}=-\left.\left(\frac{\mathrm{d} b}{\mathrm{~d} \xi} / b_{1}\right)\right|_{\xi=\xi_{p}}=\frac{b_{\mathrm{r}}-b_{\lambda}}{b_{\imath} \xi_{p}}
\end{gathered}
$$

see also [3], eqn (32)

$$
\phi_{a}=1 / \xi_{p}
$$

see also [6], eqn (23) Integration of the left parts of eqn (2) gives

$$
b=(c-1)+\left(1+a_{1}+b_{\llcorner}\right)(1-\xi)
$$

see also [3], eqns (34) and (31)

$$
\left(\phi_{a}-1\right) a_{t}+\left(\phi_{b}-1\right) b_{t}=1
$$

see also [6], eqn (33)

Equations (2')-(16) cannot be solved analytically [6] Goettler et al [2], Ouwerkerk [5] and we found an approxımate solution by linearisation of the profile of $c$, instead of using relation (15), while Ramachandran et al [6] use a linear profile for $c$ and, by maintaining eqn (15), also a linear profile for $b$

For linearisation Goettler [2] and Ramachandran[6] used the buundary conditions of (2), Ouwerkerk [5] evaluated the flux of $c$ at $\xi=\xi_{p}$ as to be the flux of $a$, while the present study matched this flux to be equal to the flux of $c$ at $\xi=1$

Table 1 summarizes the different profilc substitutions In contrast to the other authors, who assume $c=f(\xi)$ for $\xi_{p}<\xi<1$ and $c=0$ for $\xi \leqslant \xi_{p}$, Onda [4] assumes a constant value of $c$ over the whole film $0 \leq \xi \leq 1$, analogous to Van Krevelen $e t$ al [7] and Hukita et al [8] Figure 1 show the approximate profiles

\section{SOLUTIONS}

The solutions of the differential equation (2'), using (13), for models 1,2 and 3 , result in [1]

$$
\left(\frac{b_{1}}{b_{\lambda}}-1\right) \frac{1}{\xi_{p}}=\left\{\begin{array}{lll}
\frac{f\left(\lambda^{2 / 3} u\right)}{u} & \text { for } & \lambda^{2 / 3}\left(1-\xi_{p}\right) \leqslant 3 \\
0729 \lambda^{2 / 3} & \text { for } & \lambda^{2 / 3}\left(1-\xi_{p}\right)>3
\end{array}\right.
$$


Table 1 The profile substitutions

\begin{tabular}{|c|c|c|c|}
\hline & Model & Profile equation & $\begin{array}{l}\text { Profile } \\
\text { number }\end{array}$ \\
\hline $\begin{array}{l}1 \\
2 \\
3 \\
4\end{array}$ & $\begin{array}{l}\text { Goettler } \\
\text { Ouwerkerk } \\
\text { Present model } \\
\text { Onda } \\
\text { Ramachandran }\end{array}$ & 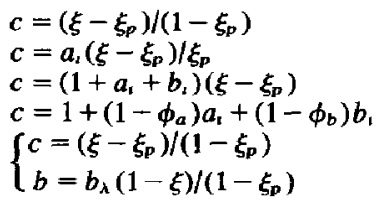 & $\begin{array}{r}1 \\
2 \\
3 \\
4 \\
\left\{\begin{array}{l}1 \\
5\end{array}\right.\end{array}$ \\
\hline
\end{tabular}

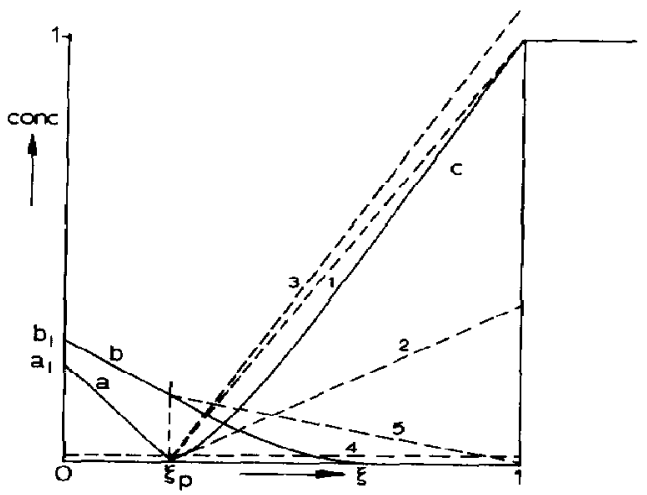

Fig 1 Film model of the simultaneous absorption of two gases, $a$ and $b$, in a reactive liquid containing $c$, one gas (a) reactung infinitely fast Dashed approximate profiles for $c$, in solving the corresponding mathematical model 1, Goettler, 2, Ouwerkerk, 3, present model, 4, Onda, $(1+5)$, Ramachandran (In fact, though not drawn, each linearisatıon method results in a different value of $\xi_{p}$ )

where

$$
\begin{gathered}
\lambda^{2}= \begin{cases}M_{b}\left(1-\xi_{p}\right) & \text { for Goettlert } \\
M_{b}\left(a_{1} / \xi_{p}\right) & \text { for Ouwerkerk } \\
M_{b}\left(1+a_{1}+b_{1}\right) & \text { for present model }\end{cases} \\
u=1-\xi_{p} \\
f\left(\lambda^{2 / 3} u\right)=\frac{1+\frac{\lambda^{2} u^{3}}{23}+\frac{\lambda^{4} u^{6}}{2356}+}{1+\frac{\lambda^{2} u^{3}}{34}+\frac{\lambda^{4} u^{6}}{3467}+}
\end{gathered}
$$

In case $a_{i}$ and $b_{b} \ll 1$, the criteria for (17a) and (17b) are equivalent with $\sqrt{ } M_{b} \leqslant 5$ and $\sqrt{ } M_{b}>5$, respectively Combination of eqns (14), (13) and (16) with eqn (17a,b) gives $\phi_{a}$ and $\phi_{b}$ (mostly an implicit system) For the other models $\phi_{a}$ and $\phi_{b}$ are also calculated from implicit equations Onda gives

$$
\phi_{a}=\frac{V\left(c M_{a}\right)}{\tanh \sqrt{ }\left(c M_{c}\right)}
$$

and

$$
\phi_{b}=\frac{\sqrt{ }\left(c M_{b}\right)}{\tanh \sqrt{ }\left(c M_{b}\right)}
$$

with $c$ given by eqn (4) in Table 1 Ramachandran calculates

$$
\phi_{b}=\frac{b_{\lambda}}{b_{i}}\left(\frac{1}{1-\xi_{p}}-\frac{M_{b}\left(1-\xi_{p}\right) b_{\lambda}}{12}\right)
$$

relatung $b_{\lambda}, \xi_{p}$ and $\phi_{a}$ by eqns (13)-(15)

\section{EVALUATION OF THE MODELS}

Startung with the model of Onda et al [4], it is evident that for $M_{a} \rightarrow \infty, c$ will approach to zero, since $\phi_{a}$ will be finite Thus the product $c M_{b}$ will be $\ll 1$ A series expansion of eqn (24) gives $\phi_{b}=1+1 / 3 c M_{b} \approx 1$ For $M_{a} \gg M_{b}$ the assumption of a constant concentration profile over the whole film may give a non-realistic model approximation, resulting in $\phi_{b}=1$ over a great range of values for $\boldsymbol{M}_{b}$

For $a_{1}$ and $b_{t} \ll 1$, the model of Ramachandran may also give big errors, because eqn (15) is used in approximating the profile of $b$ Here, two functions of $\xi$, both of high magnitude, must be subtracted in order to obtain $b$, which is of low magnitude It can be shown mathematically that in some cases enhancement factors for $b$ greater than $\sqrt{ } M_{h}$ may be calculated

From eqn (13)

$$
b_{\lambda}=b_{1}\left(1-\phi_{b} \xi_{p}\right)
$$

Elimination of $b_{\wedge}$ from (25) with eqn (26) gives

$$
\phi_{b}=\frac{T}{1+T \xi_{p}} \quad \text { with } \quad T=\frac{1}{1-\xi_{p}}+\frac{M_{b}\left(1-\xi_{p}\right)}{12}
$$

As

$$
\frac{a_{i}}{1+a_{1}} \leqslant \xi_{p} \leqslant \frac{a_{i}+b_{i}}{1+a_{1}+b_{i}}
$$

ho is evaluated as

$$
\phi_{b} \geqslant\left[\frac{12\left(1+a_{t}+b_{t}\right)}{M_{b}}+a_{t}+b_{t}\right]^{-1}
$$

For $M_{b}=1600$ and $a_{1}=b_{1}=10^{-3}$ eqn (29) gives $\phi_{b}>105$, which is greater than $\sqrt{ } M_{b}$ Figure 2 shows this once more For $10<\sqrt{ } M_{b}<120$ and $a_{1}=b_{1} / 2=250 \times 10^{-3}$ the calculated $\phi_{b}$ exceeds $\vee M_{b}$

Thus more $b$ is predicted to be absorbed in the presence of a than without $a$ This result is not in agreement with physical reality

For $a_{3} \simeq b_{1}$ the solutions obtained with the models 1,2 and 3 only differ a few percents from each other (see Fig 2) and correspond to the numerically calculated curves of Goettler et al [3]

Changing $b / a$, to, e $g$ the value of 20 , however, shows that model 2 differs markedly (up to 25\%) from the results of 1 and 3 (see Fig 3), especially for $V M_{b}>2$ and $b_{t}$ and $a_{1} \ll 1$

This could be expected from a too low assumed concentration profile of $c$, as discussed by Ouwerkerk and Goettler [9]

The present model also gives some deviations, but only for $\phi_{b}<2$ and $b_{1}>05$ For gas absorption this region is less important

An advantage of the present model over the others is that an explicit equation for $\phi_{b}$ and $\phi_{a}$ (via eqn (16)) for $V M_{b}>5$, results by elımınatıng $b_{\lambda}$

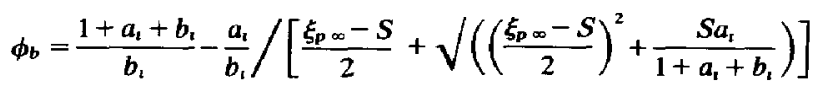

with

$$
\xi_{p \infty}=\frac{a_{i}+b_{i}}{1+a_{i}+b_{i}}
$$

$$
S=13717 /\left[\left(1+a_{1}+b_{b}\right) M_{b}\right]^{1 / 3}
$$




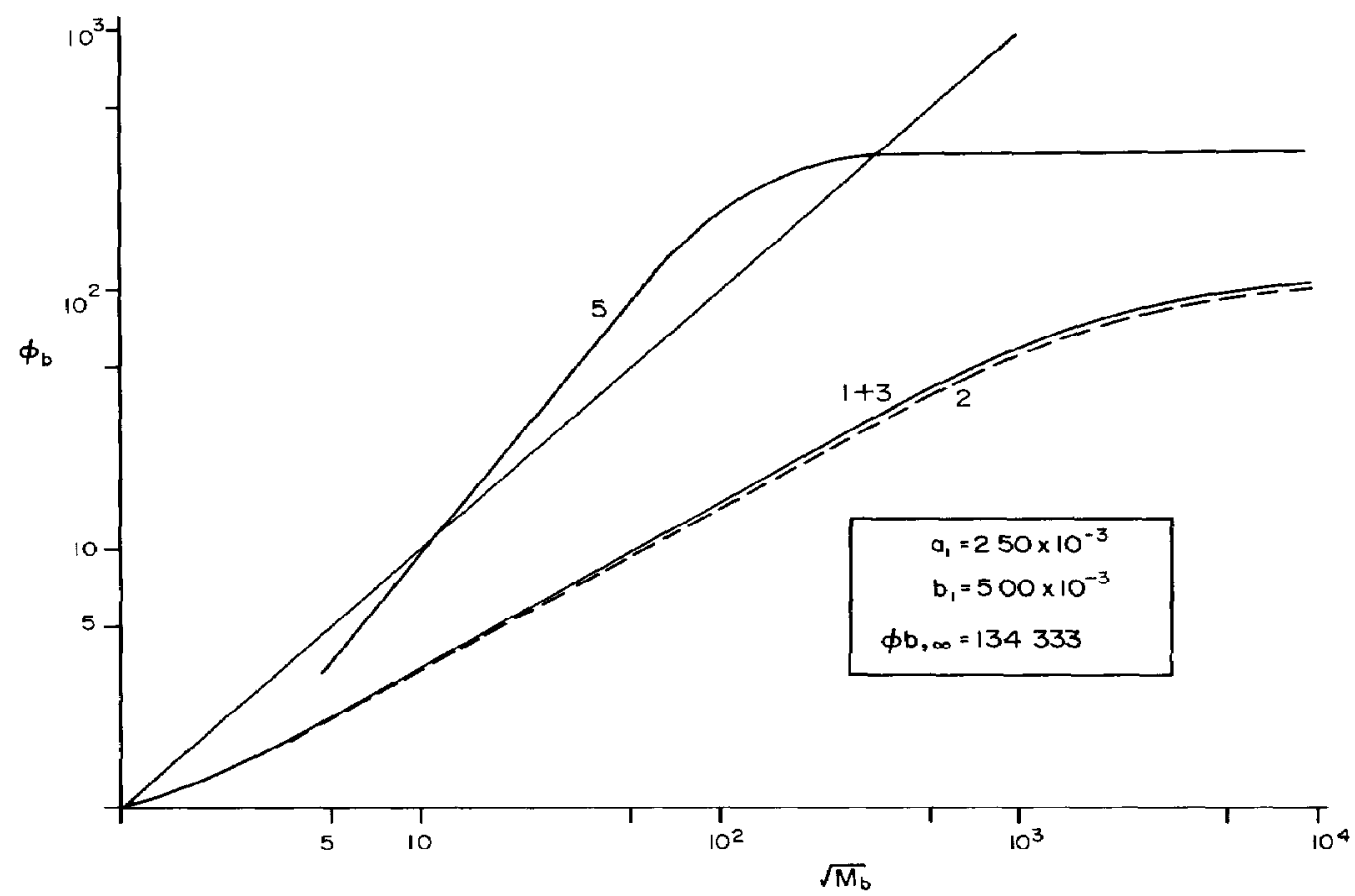

Fig 2 Plot of the enhancement factor of $b\left(\phi_{b}\right)$ vs $\vee M_{b}$ for model 1 (Goettler), 2 (Ouwerkerk), 3 (present model) and 5 (Ramachandran)

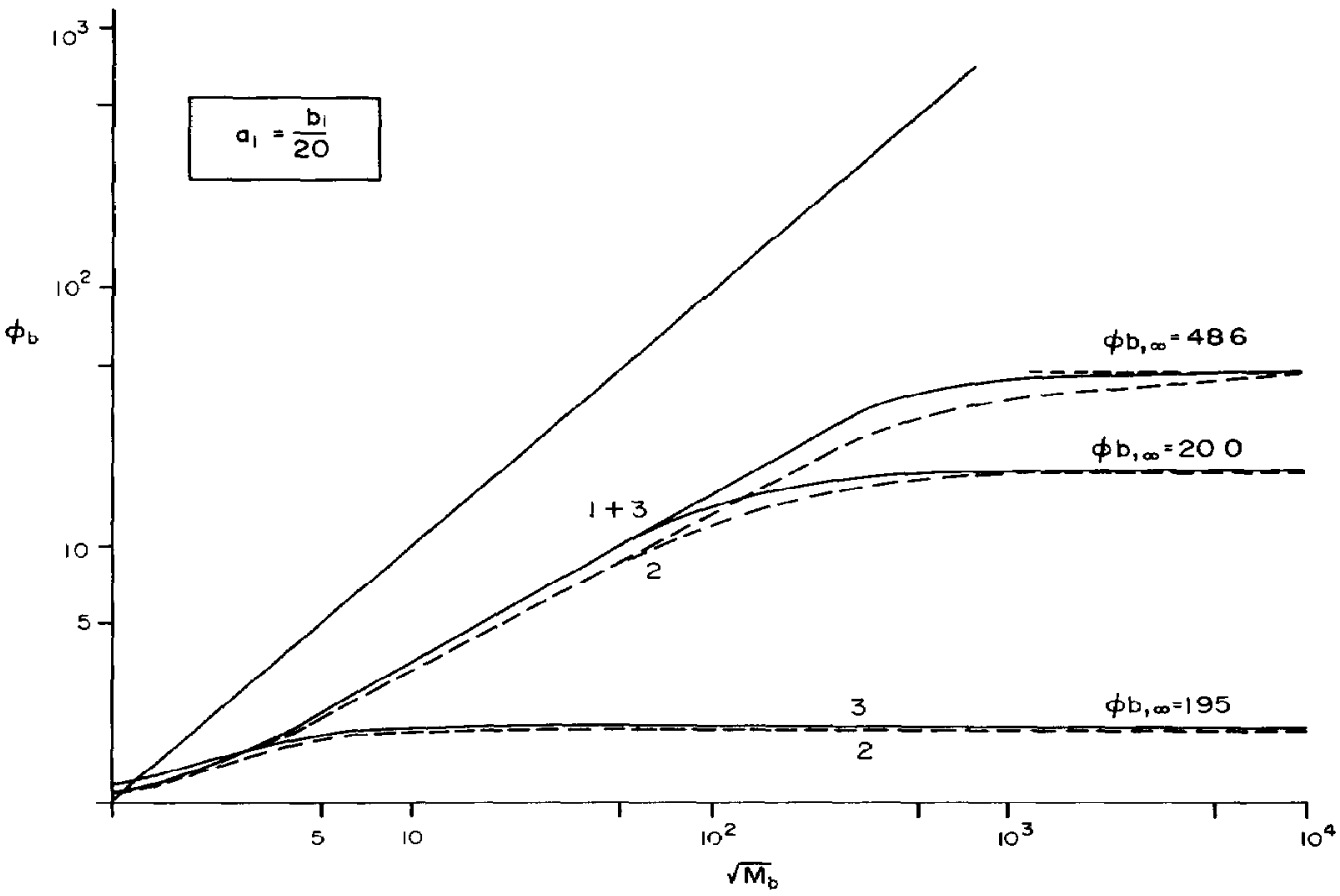

Fig 3 Plot of the enhancement factor of $b\left(\phi_{b}\right)$ vs $\sqrt{ } M_{b}$ for model 1 (Goettler), 2 (Ouwerkerk) and 3 (present model)

\section{CONCLUSIONS}

Several authors have described the simultaneous absorption of two gases, accompanied by urreversible chemical reaction, one of which is infinitely fast Two of them, [4] and [6], derived equations for the enhancement factors, which are only valud in limiting conditions

The models of Goettler et al [2], Ouwerkerk [5] and the present model are closely related, but differ somewhat for $a_{1} \ll b_{2}$

For $\sqrt{ } M_{b} \geqslant 5$, it is preferable to use our solution, which is the

only explicite one, and for $\vee M_{b}<5$ the presented extension of the Goettler model (eqn 18)

Laboratory for Chemical Reactions Engineering Department of Chemical Engineering Twente University of Technology PO Box 217 Enschede, The Netherlands
R CORNELISSE

A A C M BEENACKERS W $P$ M VAN SWAAIJ 


\section{NOTATION}

A concentration of the species entering the medrum, in which instantaneous reaction occurs, $\mathrm{kmol} / \mathrm{m}^{3}$

$a$ concentration of the species entering the medium, in dimensionless notation according to eqn (8)

$B$ concentration of the species entering the medium, in which reaction of finite rate occurs, $\mathrm{kmol} / \mathrm{m}^{3}$

$b$ concentration of the species entering the medium, in dimensionless notation according to eqn (7)

$C$ concentration of the species in the liquid, $\mathrm{kmol} / \mathrm{m}^{3}$

$c$ concentration of the species in the liquid, in dimensionless notation according to eqn (9)

$D$ diffusion coefficient, $\mathrm{m}^{2} / \mathrm{sec}$

$f()$ function of

$k$ reaction rate constant, subscript indicatıng the reaction, $\mathrm{m}^{3} /(\mathbf{k m o l ~ s e c})$

$k_{\mathrm{L}}$ physical mass transfer coefficient, $\mathrm{m} / \mathrm{sec}$

$M_{b}$ diffusion-reaction modulus of species $b$

$M_{a}$ diffusion-reaction modulus of species $a,\left(=\frac{k_{a} \delta^{2} C_{0}}{D_{a}}\right)$

$S$ dimensionless parameter defined by eqn (32)

$T$ dimensionless parameter defined by eqn (27)

$u$ dimensionless parameter defined by eqn (21)

$x$ distance in the direction of diffusion, $m$

$z$ storchiometric coefficient

Greek symbols

$\delta$ film thickness, defined by eqn (11), m

$\phi$ enhancement factor $\lambda$ distance in the film where $c=a=0, \mathrm{~m}$

$\xi$ dimensionless distance, defined by eqn (10)

Subscripts

$a, b, c$ of species $A, B, C$, respectuvely

0 in the bulk

$l$ at the interphase

$\lambda$ at $x=\lambda$

$p \quad$ at $\xi=\frac{\lambda}{\delta}$

$\infty$ in the case the reactions of $a$ and $b$ are both instantaneous

\section{REFERENCES}

[1] Abramowitz $M$ and Stegun A, Handbook of Mathematical Functions Dover, New York 1956

[2] Goettler L A and Pigford R L, I Chem E Symp Ser 1968281

[3] Goettler L A and Pigford R L, A I Ch E J 197117793

[4] Onda K, Sada E, Kobayashı $T$ and Fujıne $M$, Chem Engng Sct 1970251023

[5] Ouwerkerk C, I Chem E Symp Ser 19682839

[6] Ramachandran P A and Sharma M M, Trans Instn Chem Engrs 197149253

[7] Van Krevelen D W and Hoftızer P J, Rec Trav Chtm 194867563

[8] Hikita H and Asal S, Chem Engng Sct 196327823

[9] Discussion between Ouwerkerk C and Goettler L A, I Chem Engng Symp Ser 19682862

[10] Pangarker V G, Chem Ind Develop 1972617

Chemical Engineenng Science 1977 Vol 32 pp 1535-1536 Pergamon Press Printed in Great Britan

\title{
An improved approach of simulating chemical reactions by a Markov-chain cell model
}

\author{
(Recelved 8 August 1976, accepted 16 April 1977)
}

As reported by Pippel and Philıpp in a former publication[1] transport phenomena in chemical reaction systems can be approximately treated as stochastic processes with Markovian behaviour Especially the Markov chains, which are discrete in space and tıme (cell model), give a favourable algorithm for numerical calculations Based on these considerations a system of two matrix equations, representing the Markov approxımation of the material and energy transport, was derived However, the incorporation of kinetic expressions into these relations was done in a rather heunstic way by using a two-step procedure for each time interval $\Delta t$ (1) pure reaction step without transport and (2) transport step representing the Markov transition of matter and energy between the cells

In this paper some additional information will be given about further possibulities of handling rate expressions without integration, on the basis of the material balance in a mixing cell similar to a CSTR

$$
\frac{\mathrm{d} n_{k}}{\mathrm{~d} t}+\lambda_{\imath} n_{k}=\lambda_{1} n_{k}^{\mathrm{cn}}+v_{i} \boldsymbol{r}_{n}
$$

with the transition intensity $\lambda_{t}=v / v$

Equation (1) can be considered as a homogeneous differential equation (left part) with an inhomogeneous term $\lambda_{t} n_{k}{ }^{2 n}+v_{1} r_{k}$ The general solution for an inflow mole number $n_{k}{ }^{i n}$, which is approximately constant during $(t, t+\Delta t)$, is

$$
\begin{aligned}
n_{k}(t+\Delta t)= & n_{k}(t) \exp \left(-\lambda_{i} \Delta t\right)+n_{k}^{2 n}(t)\left[1-\exp \left(-\lambda_{i} \Delta t\right)\right] \\
& +v_{i} \exp \left(-\lambda_{i} \Delta t\right) \int_{0}^{\Delta t} r_{k}(t+s) \exp \left(\lambda_{i} s\right) \mathrm{d} s
\end{aligned}
$$

The first two terms on the RHS of eqn (2) correspond to the transition probabilittes of the Markov chains[1], simular expressions have been derived by means of the state-space method [2] They are not of further interest here

The third term in eqn (2) gives the mole number change as a result of a reaction $\Delta n_{k}{ }^{R}$

$$
\Delta n_{k}{ }^{R}=n_{t} \exp \left(-\lambda_{t} \Delta t\right) \int_{0}^{\Delta t} r_{k}(s+t) \exp (\lambda, s) d s
$$

The integral cannot be solved generally because $r_{k}$ is not exphicitely given as a function of time $t$

Some approaches have been published to calculate approximative solutions

(1) As carried out in [2], the general way of utilizing the state-space method is to set $r_{k}$ constant, 1 e $r_{k}(t+s)=r_{k}(t)$ In this case the solution of eqn (2a) is given by the expression

$$
n_{k}^{R}(t, t+\Delta t)=\frac{v_{t}}{\lambda_{1}} r_{k}(t)\left[1-\exp \left(-\lambda_{1} \Delta t\right)\right]
$$

For small changes of the reaction rate this assumption may be used However, if the rates increase considerably both changes in concentration and reaction should be taken into consideration

The only way how to guarantee the mathematical stability of the algonthm is to perform the calculation with less time steps It should be pointed out here that with eqn (3) the exact stationary states can be computed

(i1) The two-step procedure described in [1]

$$
\Delta n_{k}{ }^{R}(t, t+\Delta t)=v_{t} \exp \left(-\lambda_{t} \Delta t\right) \int_{0}^{\Delta t} r_{k}(\underline{N}(t), s) \mathrm{d} s
$$

